International Journal of Pure and Applied Mathematics

Volume 85 No. 6 2013, 1009-1019

ISSN: 1311-8080 (printed version); ISSN: 1314-3395 (on-line version)

url: http://www.ijpam.eu

doi: http://dx.doi.org/10.12732/ijpam.v85i6.4

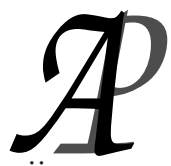

ijpam.eu

\title{
POSITIVE EQUILIBRIUM SOLUTIONS TO GENERAL POPULATION MODEL
}

\author{
Joon Hyuk Kang \\ Department of Mathematics \\ Andrews University \\ Berrien Springs, MI 49104, USA
}

Abstract: In this paper, we investigate conditions for the existence of positive solution to the following general elliptic system with various growth conditions:

$$
\left\{\begin{array}{c}
\Delta u+u(a+g(u, v))=0 \\
\Delta v+v(d+h(u, v))=0 \\
\left.u\right|_{\partial \Omega}=\left.v\right|_{\partial \Omega}=0 .
\end{array} \quad \text { in } \Omega,\right.
$$

Our arguments mainly rely on super-sub solutions, maximum principles, spectrum estimates, and some detailed properties for the solution of logistic equations.

AMS Subject Classification: 35A05, 35A07

Key Words: elliptic system with various growth conditions, existence of positive solution, super-sub solutions, maximum principles, spectrum estimates, logistic equations

\section{Introduction}

One of the prominent subjects of study and analysis in mathematical biology concerns the competition, predator-prey or cooperation of two or more species of animals in the same environment. Especially pertinent areas of investigation include the conditions under which the species can coexist, as well as the con-

Received: March 3, 2013

(c) 2013 Academic Publications, Ltd. url: www.acadpubl.eu 
ditions under which any one of the species becomes extinct, that is, one of the species is excluded by the others (see [1], [2], [3], [4], [6], [7], [11], [12], [13]). In this paper, we focus on the general population model to better understand the interactions between two species.

\section{Preliminaries}

Before entering into our primary arguments and results, we must first present a few preliminary items that we later employ throughout the proofs detailed in this paper. The following definition and lemmas are established and accepted throughout the literature on our topic.

Definition 2.1. (Super and sub solutions) Consider

$$
\left\{\begin{array}{l}
\Delta u+f(x, u)=0 \text { in } \Omega, \\
\left.u\right|_{\partial \Omega}=0
\end{array}\right.
$$

where $f \in C^{\alpha}(\bar{\Omega} \times R)$ and $\Omega$ is a bounded domain in $R^{n}$.

(A) A function $\bar{u} \in C^{2, \alpha}(\bar{\Omega})$ satisfying

$$
\left\{\begin{array}{l}
\Delta \bar{u}+f(x, \bar{u}) \leq 0 \text { in } \Omega, \\
\left.\bar{u}\right|_{\partial \Omega} \geq 0
\end{array}\right.
$$

is called a super solution to (1).

(B) A function $\underline{u} \in C^{2, \alpha}(\bar{\Omega})$ satisfying

$$
\left\{\begin{array}{l}
\Delta \underline{u}+f(x, \underline{u}) \geq 0 \text { in } \Omega, \\
\left.\underline{u}\right|_{\partial \Omega} \leq 0
\end{array}\right.
$$

is called a sub solution to (1).

Lemma 2.1. Let $f(x, \xi) \in C^{\alpha}(\bar{\Omega} \times R)$ and let $\bar{u}, \underline{u} \in C^{2, \alpha}(\bar{\Omega})$ be, respectively, super and sub solutions to (1) which satisfy $\underline{u}(x) \leq \bar{u}(x), x \in \bar{\Omega}$. Then (1) has a solution $u \in C^{2, \alpha}(\bar{\Omega})$ with $\underline{u}(x) \leq u(x) \leq \bar{u}(x), x \in \bar{\Omega}$.

In our proof, we also employ accepted conclusions concerning the solutions of the following logistic equations.

Lemma 2.2. (Established in [13]) Consider

$$
\left\{\begin{array}{l}
\Delta u+u f(u)=0 \text { in } \Omega, \\
\left.u\right|_{\partial \Omega}=0, u>0
\end{array}\right.
$$


where $f$ is a decreasing $C^{1}$ function such that there exists $c_{0}>0$ such that $f(u) \leq 0$ for $u \geq c_{0}$ and $\Omega$ is a bounded domain in $R^{n}$.

If $f(0)>\lambda_{1}$, then the above equation has a unique positive solution, where $\lambda_{1}$ is the first eigenvalue of $-\Delta$ with homogeneous boundary conditions whose corresponding eigenfunction is denoted by $\phi_{1}$. We denote this unique positive solution as $\theta_{f}$.

The most important property of this positive solution is that $\theta_{f}$ is increasing as $f$ is increasing.

We specifically note that for $a>\lambda_{1}$, the unique positive solution of

$$
\left\{\begin{array}{l}
\Delta u+u(a-u)=0 \text { in } \Omega, \\
\left.u\right|_{\partial \Omega}=0, u>0
\end{array}\right.
$$

is denoted by $\omega_{a} \equiv \theta_{a-x}$. Hence, $\theta_{a}$ is increasing as $a>0$ is increasing.

Consider the system

$$
\begin{aligned}
\Delta u+f(x, u) & =0 \text { in, } \Omega, \\
u & =0 \text { on } \partial \Omega,
\end{aligned}
$$

where $u=\left(u_{1}, \ldots, u_{m}\right)$ and $f=\left(f_{1}, \ldots, f_{m}\right)$ is quasimonotone increasing, i.e. $f_{i}(x, u)$ is increasing in $u_{j}$ for all $j \neq i$.

Lemma 2.3. ([12]) Let $w_{\lambda}$ be a family of subsolutions $(\alpha \leq \lambda \leq \beta)$ to (2), increasing in $\lambda$ such that

$$
\Delta w_{\lambda}+f\left(x, w_{\lambda}\right) \geq 0 \text { in } \Omega, w_{\lambda}=0 \text { on } \partial \Omega .
$$

Assume also $u \geq w_{\alpha}, w_{\lambda}$ does not satisfy (2) for any $\lambda$, and $\frac{\partial w_{\lambda}}{\partial n}$ changes continuously in $\lambda$ on $\partial \Omega$. Then $u \geq \sup w_{\lambda}$.

\section{Cooperating Species}

In [12], Korman and Leung established a sufficient and necessary condition for the existence of positive solution to the cooperation system

$$
\left\{\begin{array}{c}
\Delta u(x)+u(x)(a-u(x)+c v(x))=0 \\
\Delta v(x)+v(x)(d-v(x)+e u(x))=0 \\
\left.u(x)\right|_{\partial \Omega}=\left.v(x)\right|_{\partial \Omega}=0 .
\end{array}\right.
$$

where $\Omega$ is a bounded domain in $R^{n}, u(x)$ and $v(x)$ designate the population densities for the two species.

The following is their result: 
Theorem 3.1. For existence of a positive solution to (3) it is necessary and sufficient that $c e<1$.

In this section, we develop their result to more general population model:

$$
\left\{\begin{array}{c}
\Delta u(x)+u(x)(a+g(u(x), v(x)))=0 \\
\Delta v(x)+v(x)(d+h(u(x), v(x)))=0 \\
\left.u\right|_{\partial \Omega}=\left.v\right|_{\partial \Omega}=0
\end{array}\right.
$$

where $a$ and $d$ are positive constants, $g, h \in C^{1}$ are such that $g_{u}<0, g_{v}>$ $0, h_{u}>0, h_{v}<0$ and $g(0,0)=h(0,0)=0$.

The following Theorem proves a necessary condition for the existence of a positive solution to (4).

Theorem 3.2. If $a>\lambda_{1}, d>\lambda_{1}, \inf \left(h_{v}\right) \geq-1$, and $\inf \left(h_{u}\right)>0$, then the existence of a positive solution to (4) implies

$$
\inf \left(g_{v}\right) \inf \left(h_{u}\right)+\inf \left(g_{u}\right)<0 .
$$

Proof. Suppose $\inf \left(g_{v}\right) \inf \left(h_{u}\right)+\inf \left(g_{u}\right) \geq 0$. Consider a family $\left(u_{\lambda}, v_{\lambda}\right)=$ $\left(\lambda \phi_{1}, \lambda \inf \left(h_{u}\right) \phi_{1}\right)$ with any $\lambda>0$.

Then by the assumption and Mean Value Theorem,

$$
\begin{aligned}
& \Delta u_{\lambda}+u_{\lambda}\left[a+g\left(u_{\lambda}, v_{\lambda}\right)\right] \\
= & -\lambda \lambda_{1} \phi_{1}+\lambda \phi_{1}\left[a+g\left(\lambda \phi_{1}, \lambda \inf \left(h_{u}\right) \phi_{1}\right)\right] \\
= & \lambda \phi_{1}\left[-\lambda_{1}+a+g\left(\lambda \phi_{1}, \lambda \inf \left(h_{u}\right) \phi_{1}\right)\right] \\
= & \lambda \phi_{1}\left[a-\lambda_{1}+g\left(\lambda \phi_{1}, \lambda \inf \left(h_{u}\right) \phi_{1}-g\left(\lambda \phi_{1}, 0\right)+g\left(\lambda \phi_{1}, 0\right)-g(0,0)\right]\right. \\
\geq & \lambda \phi_{1}\left[a-\lambda_{1}+\inf \left(g_{v}\right) \inf \left(h_{u}\right) \lambda \phi_{1}+\inf \left(g_{u}\right) \lambda \phi_{1}\right] \\
> & 0,
\end{aligned}
$$

and

$$
\begin{aligned}
& \Delta v_{\lambda}+v_{\lambda}\left[d+h\left(u_{\lambda}, v_{\lambda}\right)\right] \\
= & -\lambda \inf \left(h_{u}\right) \lambda_{1} \phi_{1}+\lambda \inf \left(h_{u}\right) \phi_{1}\left[d+h\left(\lambda \phi_{1}, \lambda \inf \left(h_{u}\right) \phi_{1}\right)\right] \\
= & \lambda \inf \left(h_{u}\right) \phi_{1}\left[-\lambda_{1}+d+h\left(\lambda \phi_{1}, \lambda \inf \left(h_{u}\right) \phi_{1}\right)\right] \\
= & \lambda \inf \left(h_{u}\right) \phi_{1}\left[d-\lambda_{1}+h\left(\lambda \phi_{1}, \lambda \inf \left(h_{u}\right) \phi_{1}\right)-h\left(\lambda \phi_{1}, 0\right)+h\left(\lambda \phi_{1}, 0\right)\right. \\
& -h(0,0)] \\
\geq & \lambda \inf \left(h_{u}\right) \phi_{1}\left[d-\lambda_{1}+\inf \left(h_{v}\right) \lambda \inf \left(h_{u}\right) \phi_{1}+\inf \left(h_{u}\right) \lambda \phi_{1}\right] \\
> & 0 .
\end{aligned}
$$

Therefore, $\left(u_{\lambda}, v_{\lambda}\right)=\left(\lambda \phi_{1}, \lambda \inf \left(h_{u}\right) \phi_{1}\right)$ with any $\lambda>0$ is a family of subsolutions to (4). 
Furthermore, if $(u, v)$ is a positive solution to (4), then $u>\lambda_{0} \phi_{1}$ and $v>\lambda_{0} \inf \left(h_{u}\right) \phi_{1}$ for suffuciently small $\lambda_{0}>0$, and so by the lemma 2.3, we conclude that $u \geq \lambda \phi_{1}$ and $v \geq \lambda \inf \left(h_{u}\right) \phi_{1}$ for any $\lambda \geq \lambda_{0}$.

Hence, there is no positive solution to (4).

For a sufficient condition for the existence of a positive solution to (4), we need the following Lemma.

Lemma 3.3. If bf $>c e$, then we can choose arbitrary large $M, N>0$ such that

$$
\begin{aligned}
& a-b M+c N<0, \\
& d+e u-f N<0 .
\end{aligned}
$$

We now establish a sufficient condition for the existence of a positive solution to $(4)$.

Theorem 3.4. If $a>\lambda_{1}, d>\lambda_{1}$ and $\sup \left(g_{v}\right) \sup \left(h_{u}\right)<\sup \left(g_{u}\right) \sup \left(h_{v}\right)$, then (4) has a positive solution.

Proof. Let $\underline{u}=\alpha \phi_{1}, \underline{v}=\beta \phi_{1}$, where $\alpha, \beta>0$.

Then since $a>\lambda_{1}$ and $d>\lambda_{1}$, by the Mean Value Theorem, for small enough $\alpha, \beta>0$,

$$
\begin{aligned}
& \Delta \underline{u}+\underline{u}[a+g(\underline{u}, \underline{v})] \\
= & -\alpha \lambda_{1} \phi_{1}+\alpha \phi_{1}\left[a+g\left(\alpha \phi_{1}, \beta \phi_{1}\right)\right] \\
= & \alpha \phi_{1}\left[-\lambda_{1}+a+g\left(\alpha \phi_{1}, \beta \phi_{1}\right)\right] \\
= & \alpha \phi_{1}\left[-\lambda_{1}+a+g\left(\alpha \phi_{1}, \beta \phi_{1}\right)-g\left(0, \beta \phi_{1}\right)+g\left(0, \beta \phi_{1}\right)-g(0,0)\right] \\
\geq & \alpha \phi_{1}\left[-\lambda_{1}+a+\inf \left(g_{u}\right) \alpha \phi_{1}+\inf \left(g_{v}\right) \beta \phi_{1}\right] \\
\geq & 0,
\end{aligned}
$$

and

$$
\begin{aligned}
& \Delta \underline{v}+\underline{v}[d+h(\underline{u}, \underline{v})] \\
= & -\beta \lambda_{1} \phi_{1}+\beta \phi_{1}\left[d+h\left(\alpha \phi_{1}, \beta \phi_{1}\right)\right] \\
= & \beta \phi_{1}\left[-\lambda_{1}+d+h\left(\alpha \phi_{1}, \beta \phi_{1}\right)\right] \\
= & \beta \phi_{1}\left[-\lambda_{1}+d+h\left(\alpha \phi_{1}, \beta \phi_{1}\right)-h\left(0, \beta \phi_{1}\right)+h\left(0, \beta \phi_{1}\right)-h(0,0)\right] \\
\geq & \beta \phi_{1}\left[-\lambda_{1}+d+\inf \left(h_{u}\right) \alpha \phi_{1}+\inf \left(h_{v}\right) \beta \phi_{1}\right] \\
\geq & 0
\end{aligned}
$$

and so, $(\underline{u}, \underline{v})=\left(\alpha \phi_{1}, \beta \phi_{1}\right)$ is a subsolution to (4) for sufficiently small $\alpha, \beta>0$.

But, for all $(u, v)$, by the Mean Value Theorem again,

$$
\begin{aligned}
g(u, v) & =g(u, v)-g(u, 0)+g(u, 0)-g(0,0) \\
& \leq \sup \left(g_{v}\right) v+\sup \left(g_{u}\right) u
\end{aligned}
$$


and

$$
\begin{aligned}
h(u, v) & =h(u, v)-h(u, 0)+h(u, 0)-h(0,0) \\
& \leq \sup \left(h_{v}\right) v+\sup \left(h_{u}\right) u,
\end{aligned}
$$

so by the condition and the Lemma 3.3, there are constants $M, N>0$ with $\alpha \phi_{1}<M, \beta \phi_{1}<N$ such that

$$
\begin{aligned}
& \Delta M+M[a+g(M, N)] \leq M\left[a+\sup \left(g_{v}\right) N+\sup \left(g_{u}\right) M\right]<0, \\
& \Delta N+N[d+h(M, N)] \leq N\left[d+\sup \left(h_{v}\right) N+\sup \left(h_{u}\right) M\right]<0,
\end{aligned}
$$

in other words, $(M, N)$ is a supersolution to (4).

We conclude by the Lemma 2.1 that there is a positive solution $(u, v)$ to $(4)$ with $\alpha \phi_{1} \leq u \leq M, \beta \phi_{1} \leq v \leq N$.

\section{Competing Species}

In [4], Cosner and Lazer established a sufficient and necessary condition for the existence of positive solution to the competing system

$$
\left\{\begin{aligned}
\Delta u(x)+u(x)(a-b u(x)-c v(x))=0 \\
\Delta v(x)+v(x)(a-f v(x)-e u(x))=0 \\
\left.u(x)\right|_{\partial \Omega}=\left.v(x)\right|_{\partial \Omega}=0
\end{aligned} \quad \text { in } \Omega,\right.
$$

where $\Omega$ is a bounded domain in $R^{n}, a, b, c, e, f>0$ are constants, $u(x)$ and $v(x)$ designate the population densities for the two species.

The following is their result:

Theorem 4.1. In order that there exist positive smooth functions $u$ and $v$ in $\Omega$ satisfying (5), it is necessary and sufficient that one of the following three sets of conditions holds.

$$
\begin{aligned}
& (i) a>\lambda_{1}, b>e, c<f \\
& \left(\text { ii } a>\lambda_{1}, b=e, c=f\right. \\
& \left(\text { iii } a>\lambda_{1}, b<e, c>f\right.
\end{aligned}
$$

In this section, we develop their result to more general population model:

$$
\left\{\begin{aligned}
\Delta u(x)+u(x)[a+g(u(x), v(x))] & =0 \quad \text { in } \Omega, \\
\Delta v(x)+v(x)[a+h(u(x), v(x))] & =0 \\
\left.u\right|_{\partial \Omega}=\left.v\right|_{\partial \Omega}=0 &
\end{aligned}\right.
$$

where $a$ is a positive constant, $g, h \in C^{1}$ are such that $g_{u}<0, g_{v}<0, h_{u}<$ $0, h_{v}<0$, there exist constants $c_{0}>0, c_{1}>0$ such that $a+g(u, 0) \leq 0$ for $u \geq c_{0}$ and $a+h(0, v) \leq 0$ for $v \geq c_{1}$, and $g(0,0)=h(0,0)=0$. 
The following theorem provides a sufficient condition for the existence of a positive smooth solution to (6).

Theorem 4.2. Suppose one of the following three sets of conditions holds.

$$
\begin{aligned}
& (1) a>\lambda_{1}, \inf \left(g_{u}\right)<\inf \left(h_{u}\right), \inf \left(g_{v}\right)>\inf \left(h_{v}\right) \\
& (2) a>\lambda_{1}, \inf \left(g_{u}\right)=\inf \left(h_{u}\right), \inf \left(g_{v}\right)=\inf \left(h_{v}\right) \\
& (3) a>\lambda_{1}, \inf \left(g_{u}\right)>\inf \left(h_{u}\right), \inf \left(g_{v}\right)<\inf \left(h_{v}\right)
\end{aligned}
$$

Then (6) has a positive smooth solution.

Proof. By the Theorem 4.1, if one of the above three sets of conditions holds, then there is a positive smooth solution $(u, v)$ to

$$
\begin{aligned}
& \Delta u+u\left[a-\left(-\inf \left(g_{u}\right)\right) u-\left(-\inf \left(g_{v}\right)\right) v\right]=0 \\
& \Delta v+v\left[a-\left(-\inf \left(h_{u}\right)\right) u-\left(-\inf \left(h_{v}\right)\right) v\right]=0
\end{aligned} \quad \text { in } \Omega,\left.u\right|_{\partial \Omega}=\left.v\right|_{\partial \Omega}=0 .
$$

But, by the Mean Value Theorem,

$$
\begin{aligned}
& \Delta u+u[a+g(u, v)] \\
= & \Delta u+u[a+g(u, v)-g(0, v)+g(0, v)-g(0,0)] \\
\geq & \Delta u+u\left[a+\inf \left(g_{u}\right) u+\inf \left(g_{v}\right) v\right] \\
= & \Delta u+u\left[a-\left(-\inf \left(g_{u}\right)\right) u-\left(-\inf \left(g_{v}\right)\right) v\right] \\
= & 0
\end{aligned}
$$

and

$$
\begin{aligned}
& \Delta v+v[a+h(u, v)] \\
= & \Delta v+v[a+h(u, v)-h(0, v)+h(0, v)-h(0,0)] \\
\geq & \Delta v+v\left[a+\inf \left(h_{u}\right) u+\inf \left(h_{v}\right) v\right] \\
= & \Delta v+v\left[a-\left(-\inf \left(h_{u}\right)\right) u-\left(-\inf \left(h_{v}\right)\right) v\right] \\
= & 0 .
\end{aligned}
$$

Hence, $(u, v)$ is a subsolution to (6).

But by the conditions of $g, h$, any large positive constant $M$ satisfying $u<$ $M, v<M$ in $\Omega$ is a supersolution to (6).

Therefore, by the Lemma 2.1, (6) has a positive smooth solution.

The next theorem establishes a necessary condition for the existence of a positive smooth solution to (6).

Theorem 4.3. If (6) has a positive smooth solution, then $a>\lambda_{1}$ and one 
of the following six sets of conditions holds.

(1) $g_{u} \equiv h_{u}$ are constants, $\inf \left(h_{v}\right) \leq \sup \left(g_{v}\right), \sup \left(h_{v}\right) \geq \inf \left(g_{v}\right)$

(2) $\inf \left(h_{u}\right)=\sup \left(g_{u}\right), \sup \left(h_{u}\right)>\inf \left(g_{u}\right), \inf \left(h_{v}\right) \leq \sup \left(g_{v}\right)$

(3) $\inf \left(h_{u}\right)>\sup \left(g_{u}\right), \sup \left(h_{u}\right)>\inf \left(g_{u}\right), \inf \left(h_{v}\right)<\sup \left(g_{v}\right)$

(4) $\inf \left(h_{u}\right)<\sup \left(g_{u}\right), \sup \left(h_{u}\right)=\inf \left(g_{u}\right), \sup \left(h_{v}\right) \geq \inf \left(g_{v}\right)$

(5) $\inf \left(h_{u}\right)<\sup \left(g_{u}\right), \sup \left(h_{u}\right)<\inf \left(g_{u}\right), \sup \left(h_{v}\right)>\inf \left(g_{v}\right)$

(6) $\inf \left(h_{u}\right)<\sup \left(g_{u}\right), \sup \left(h_{u}\right)>\inf \left(g_{u}\right)$

Proof. Suppose $(u, v)$ is a positive smooth solution to (6).

By the Mean Value Theorem, there are $\tilde{u}, \tilde{v}$ with $0 \leq \tilde{u} \leq u, 0 \leq \tilde{v} \leq v$ such that

$$
\begin{aligned}
& g(u, 0)-g(0,0)=g_{u}(\tilde{u}, 0) u \\
& g(u, v)-g(u, 0)=g_{v}(u, \tilde{v}) v
\end{aligned}
$$

Hence, by the Green's Identity,

$$
\begin{aligned}
& \int_{\Omega} u \phi_{1}\left[\lambda_{1}-a-g_{u}(\tilde{u}, 0) u-g_{v}(u, \tilde{v}) v\right] d x \\
= & \int_{\Omega} u \phi_{1}\left[\lambda_{1}-a+g(0,0)-g(u, 0)+g(u, 0)-g(u, v)\right] d x \\
= & \int_{\Omega} u \phi_{1}\left[\lambda_{1}-a-g(u, v)\right] d x \\
= & \int_{\Omega} \phi_{1}[-a u-u g(u, v)]+u \lambda_{1} \phi_{1} d x \\
= & \int_{\Omega} \phi_{1} \Delta u-u \Delta \phi_{1} d x \\
= & 0 .
\end{aligned}
$$

But, since $-g_{u}(\tilde{u}, 0) u-g_{v}(u, \tilde{v}) v>0$ in $\Omega, a>\lambda_{1}$.

By the Mean Value Theorem again, there are $u_{1}, u_{2}, v_{1}, v_{2}$ with $0 \leq u_{1}, u_{2} \leq$ $u, 0 \leq v_{1}, v_{2} \leq v$ such that

$$
\begin{aligned}
& g(u, v)-g(0, v)=g_{u}\left(u_{1}, v\right) u, \\
& h(u, v)-h(0, v)=h_{u}\left(u_{2}, v\right) u, \\
& g(0, v)-g(0,0)=g_{v}\left(0, v_{1}\right) v, \\
& h(0, v)-h(0,0)=h_{v}\left(0, v_{2}\right) v .
\end{aligned}
$$

Therefore, by the Green's Identity again,

$$
\begin{aligned}
& \int_{\Omega} u v\left(\left[h_{u}\left(u_{2}, v\right)-g_{u}\left(u_{1}, v\right)\right] u+\left[h_{v}\left(0, v_{2}\right)-g_{v}\left(0, v_{1}\right)\right] v\right) d x \\
= & \int_{\Omega} u v\left[h_{u}\left(u_{2}, v\right) u+h_{v}\left(0, v_{2}\right) v-g_{u}\left(u_{1}, v\right) u-g_{v}\left(0, v_{1}\right) v\right] d x \\
= & \int_{\Omega} u v[h(u, v)-h(0, v)+h(0, v)-h(0,0)+g(0, v)-g(u, v)-g(0, v) \\
& +g(0,0)] d x \\
= & \int_{\Omega} u v[h(u, v)-g(u, v)] d x \\
= & \int_{\Omega} v \Delta u-u \Delta v d x \\
= & 0
\end{aligned}
$$


and so,

$$
\begin{aligned}
& \int_{\Omega} u v\left(\left[\inf \left(h_{u}\right)-\sup \left(g_{u}\right)\right] u+\left[\inf \left(h_{v}\right)-\sup \left(g_{v}\right)\right] v\right) d x \leq 0, \\
& \int_{\Omega} u v\left(\left[\sup \left(h_{u}\right)-\inf \left(g_{u}\right)\right] u+\left[\sup \left(h_{v}\right)-\inf \left(g_{v}\right)\right] v\right) d x \geq 0,
\end{aligned}
$$

which derives

$$
\begin{aligned}
& (A) \inf \left(h_{u}\right)=\sup \left(g_{u}\right), \inf \left(h_{v}\right) \leq \sup \left(g_{v}\right), \\
& (B) \inf \left(h_{u}\right)>\sup \left(g_{u}\right), \inf \left(h_{v}\right)<\sup \left(g_{v}\right), \\
& (C) \inf \left(h_{u}\right)<\sup \left(g_{u}\right),
\end{aligned}
$$

and

$$
\begin{aligned}
& \left(A^{\prime}\right) \sup \left(h_{u}\right)=\inf \left(g_{u}\right), \sup \left(h_{v}\right) \geq \inf \left(g_{v}\right), \\
& \left(B^{\prime}\right) \sup \left(h_{u}\right)<\inf \left(g_{u}\right), \sup \left(h_{v}\right)>\inf \left(g_{v}\right), \\
& \left(C^{\prime}\right) \sup \left(h_{u}\right)>\inf \left(g_{u}\right) .
\end{aligned}
$$

Combining $(A),(B),(C)$ and $\left(A^{\prime}\right),\left(B^{\prime}\right),\left(C^{\prime}\right)$ together, we may have

$\left(A^{\prime \prime}\right) g_{u} \equiv h_{u}$ are constants, $\inf \left(h_{v}\right) \leq \sup \left(g_{v}\right), \sup \left(h_{v}\right) \geq \inf \left(g_{v}\right)$,

$\left(B^{\prime \prime}\right) \inf \left(h_{u}\right)=\sup \left(g_{u}\right), \sup \left(h_{u}\right)<\inf \left(g_{u}\right), \inf \left(h_{v}\right) \leq \sup \left(g_{v}\right), \sup \left(h_{v}\right)>\inf \left(g_{v}\right)$, $\left(C^{\prime \prime}\right) \inf \left(h_{u}\right)=\sup \left(g_{u}\right), \sup \left(h_{u}\right)>\inf \left(g_{u}\right), \inf \left(h_{v}\right) \leq \sup \left(g_{v}\right)$,

$\left(D^{\prime \prime}\right) \inf \left(h_{u}\right)>\sup \left(g_{u}\right), \sup \left(h_{u}\right)=\inf \left(g_{u}\right), \inf \left(h_{v}\right)<\sup \left(g_{v}\right), \sup \left(h_{v}\right) \geq \inf \left(g_{v}\right)$,

$\left(E^{\prime \prime}\right) \inf \left(h_{u}\right)>\sup \left(g_{u}\right), \sup \left(h_{u}\right)<\inf \left(g_{u}\right), \inf \left(h_{v}\right)<\sup \left(g_{v}\right), \sup \left(h_{v}\right)>\inf \left(g_{v}\right)$,

$\left(F^{\prime \prime}\right) \inf \left(h_{u}\right)>\sup \left(g_{u}\right), \sup \left(h_{u}\right)>\inf \left(g_{u}\right), \inf \left(h_{v}\right)<\sup \left(g_{v}\right)$,

$\left(G^{\prime \prime}\right) \inf \left(h_{u}\right)<\sup \left(g_{u}\right), \sup \left(h_{u}\right)=\inf \left(g_{u}\right), \sup \left(h_{v}\right) \geq \inf \left(g_{v}\right)$,

$\left(H^{\prime \prime}\right) \inf \left(h_{u}\right)<\sup \left(g_{u}\right), \sup \left(h_{u}\right)<\inf \left(g_{u}\right), \sup \left(h_{v}\right)>\inf \left(g_{v}\right)$,

(I") $\inf \left(h_{u}\right)<\sup \left(g_{u}\right), \sup \left(h_{u}\right)>\inf \left(g_{u}\right)$.

However, it is clear that $\left(B^{\prime \prime}\right),\left(D^{\prime \prime}\right),\left(E^{\prime \prime}\right)$ are not possible, so we establish the result of the Theorem.

Finally, we prove a nonexistence result.

Theorem 4.4. If $a>\frac{\nu}{\mu} d,-1 \leq g_{u}<0$, and $h_{v} \leq-1$, where $\mu=$ $\min \left[-\sup \left(h_{u}\right), 1\right]$ and $\nu=\max \left[-\inf \left(g_{v}\right), 1\right]$, then there is no positive solution to $(6)$.

Proof. Suppose there is a positive solution $(u, v)$ to $(6)$.

Then by the Mean Value Theorem, the Green's Identity and the inequality 
conditions,

$$
\begin{aligned}
& \int_{\Omega}\left(a-d+\left[-\sup \left(h_{u}\right)-1\right] u+\left[1+\inf \left(g_{v}\right)\right] v\right) u v d x \\
\leq & \int_{\Omega}\left(a-d+\left[\inf \left(g_{u}\right)-\sup \left(h_{u}\right)\right] u+\left[\inf \left(g_{v}\right)-\sup \left(h_{v}\right)\right] v\right) u v d x \\
\leq & \int_{\Omega}[a-d+g(u, 0)-g(0,0)-h(u, v)+h(0, v)+g(u, v)-g(u, 0)-h(0, v) \\
& +h(0,0)] u v d x \\
= & \int_{\Omega}[a-d+g(u, v)-h(u, v)] u v d x \\
= & \int_{\Omega}(v \Delta u-u \Delta v) d x \\
= & 0 .
\end{aligned}
$$

But, if $a>\frac{\nu}{\mu} d$, then since $a \geq u$ and $d \geq v$,

$$
a-d+\left[-\sup \left(h_{u}\right)-1\right] u+\left[1+\inf \left(g_{v}\right)\right] v \geq \mu a-\nu d>0,
$$

which contradicts to $(7)$.

\section{References}

[1] S.W. Ali, C. Cosner, On the uniqueness of the positive steady state for Lotka-Volterra Models with diffusion, Journal of Mathematical Analysis and Application, 168 (1992), 329-341.

[2] R.S. Cantrell, C. Cosner, On the steady-state problem for the VolterraLotka competition model with diffusion, Houston Journal of Mathematics, 13 (1987), 337-352.

[3] R.S. Cantrell, C. Cosner, On the uniqueness and stability of positive solutions in the Volterra-Lotka competition model with diffusion, Houston J. Math., 15 (1989), 341-361.

[4] C. Cosner, A.C. Lazer, Stable coexistence states in the Volterra-Lotka competition model with diffusion, Siam J. Appl. Math., 44 (1984), 11121132.

[5] D. Dunninger, Lecture Note for Applied Analysis in Michigan State University.

[6] J. L.-Gomez, R. Pardo, Existence and uniqueness for some competition models with diffusion, C.R. Acad. Sci. Paris, Ser. I Math., 313 (1991), 933-938. 
[7] C. Gui, Y. Lou, Uniqueness and nonuniqueness of coexistence states in the Lotka-Volterra competition model, Comm Pure and Appl. Math., XVL2, No. 12 (1994), 1571-1594.

[8] J. Kang, Y. Oh, A sufficient condition for the uniqueness of positive steady state to a reaction diffusion system, Journal of Korean Mathematical Society, 39, No. 39 (2002), 377-385.

[9] J. Kang, Y. Oh, Uniqueness of coexistence state of general competition model for several competing species, Kyungpook Mathematical Journal, 42, No. 2 (2002), 391-398.

[10] J. Kang, Y. Oh, J. Lee, The existence, nonexistence and uniqueness of global positive coexistence of a nonlinear elliptic biological interacting model, Kangweon-Kyungki Math. Jour., 12, No. 1 (2004), 77-90.

[11] P. Korman, A. Leung, A general monotone scheme for elliptic systems with applications to ecological models, Proceedings of the Royal Society of Edinburgh, 102A (1986), 315-325

[12] P. Korman, A. Leung, On the existence and uniqueness of positive steady states in the Volterra-Lotka ecological models with diffusion, Applicable Analysis, 26, 145-160.

[13] L. Li, R. Logan, Positive solutions to general elliptic competition models, Differential and Integral Equations, 4 (1991), 817-834.

[14] A. Leung, Equilibria and stabilities for competing-species, reactiondiffusion equations with Dirichlet boundary data, J. Math. Anal. Appl., 73 (1980), 204-218. 
